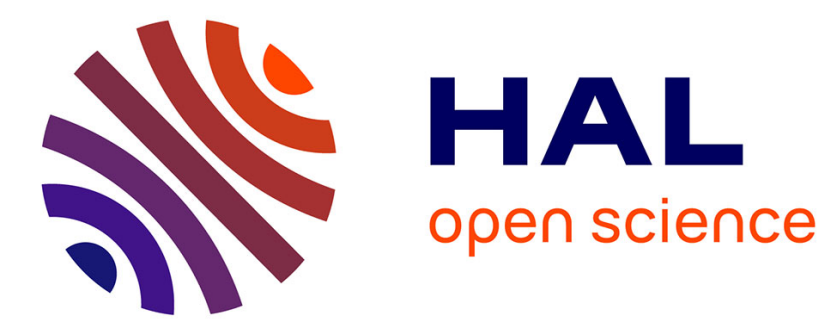

\title{
La fin des cadres de classement
}

Bénédicte Grailles

\section{To cite this version:}

Bénédicte Grailles. La fin des cadres de classement. 2009. halshs-00371213

\section{HAL Id: halshs-00371213 \\ https://shs.hal.science/halshs-00371213}

Preprint submitted on 26 Mar 2009

HAL is a multi-disciplinary open access archive for the deposit and dissemination of scientific research documents, whether they are published or not. The documents may come from teaching and research institutions in France or abroad, or from public or private research centers.
L'archive ouverte pluridisciplinaire HAL, est destinée au dépôt et à la diffusion de documents scientifiques de niveau recherche, publiés ou non, émanant des établissements d'enseignement et de recherche français ou étrangers, des laboratoires publics ou privés. 


\title{
Centre de recherches historiques de l'Ouest (CERHIO-UMR CNRS 6258)
}

\author{
Site d'Angers
}

\section{Séminaire ALMA 2008-2009 : Les raisons classificatoires}

Séance du 26 février 2009

\section{La fin des cadres de classement}

\section{Bénédicte Grailles (CERHIO-UMR CNRS 6258)}

Le cadre de classement a été, pendant un peu plus d'un siècle et demi, l'instrument privilégié de l'accès aux fonds d'archives publiques en France. Plan directeur préétabli qui fixe au sein d'un service d'archives la répartition des fonds et des collections en de grandes divisions et subdivisions méthodiques - séries et sous-séries -, il constitue le plan général de répartition alphanumérique des documents conservés dans ce service, établi en fonction de différents critères, afin de faciliter la recherche de ces documents. Savants mélanges entre une répartition par fonds et une répartition méthodique - par thème - au niveau des grandes fonctions administratives, compromis entre «principe de provenance » et «principe de pertinence ${ }^{1}$, les cadres de classement les plus efficients - commune et département - datent du milieu du XIX ${ }^{\mathrm{e}}$ siècle $^{2}$. La question de la clôture des cadres de classement, c'est-à-dire de l'adoption d'un nouveau système de répartition des fonds, est centrale dans la pratique archivistique française et constitue une révolution dans les techniques mises en œuvre pour le traitement matériel et intellectuel des documents. Nous nous intéresserons essentiellement à une période charnière, celle allant des années 1950 aux années 1980. La question du cadre de classement est liée à celle - plus générale - des archives contemporaines.

Après la seconde guerre mondiale, les archivistes sont contraints de s'interroger sur les documents contemporains, leur croissance exponentielle, leur aspect sériel, la mauvaise tenue des dossiers d'affaire, la multiplication des doubles, mais aussi sur les modes de classement dans les services administratifs avec l'apparition de nouveaux matériels comme les meubles à clapets ou rotatifs, et sur les techniques d'automatisation comme les fiches mécanographiques. Ces changements d'échelle, de nature, de volume et de qualité des

1. Pour reprendre les termes utilisés dans la circulaire de la direction des Archives de France AD 79-6 du 31 décembre 1979. Le principe de provenance est une des composantes du respect des fonds. Le respect des fonds implique le respect de la provenance, le respect de l'intégrité du fonds et, lorsque cela est possible, le respect de l'ordre originel ou primitif au cours du classement.

2. I1 existe des cadres de classement réglementaires pour les Archives nationales, archives départementales, archives communales et hospitalières. Ces cadres de classement avaient, mis à part celui des Archives nationales initié par Daunou et entaché, dès l'origine, d'un vice de conception pour avoir provoqué un démembrement des fonds, rempli leur office depuis 1841 pour celui des archives départementales et 1842 pour les archives communales, avec des adaptations régulières (fig. 1). 
documents ébranlent les certitudes professionnelles. Concurremment, les échanges internationaux se multiplient avec les créations du Conseil international des archives fondé en 1948 dans la foulée de 1'Unesco et du stage technique international d'archives initié par la direction des Archives de France en 1951. Le chartiste Yves Pérotin importe dans la sphère française la théorie des trois âges conceptualisé par l'Américain Th. Shellenberg et des techniques anglo-saxonnes.

Le malaise des archivistes français se cristallise autour de la question du cadre de classement. Les cadres de classement, s'ils s'avèrent relativement adaptés au classement externe des fonds clos, posent de nombreux problèmes pour les fonds ouverts, susceptibles d'accroissements, au sein desquels des éliminations devront être pratiquées et qui sont régulièrement consultés par les services qui les ont produits. En particulier, à une époque où les services d'archives ne disposent d'aucune aide automatisée pour tenir à jour un récolement permanent, les séries obligent à réserver des espaces pour permettre les accroissements. Comme parallèlement certains services d'archives départementales, au demeurant pas majoritaires, s'orientent vers une politique active de pré-archivage, le problème est accentué par la question connexe de la gestion des éliminables, amenant progressivement à leur réserver des magasins : c'est la pratique dite de la « salle d'attente ».

La question centrale est celle des intercalations de dossiers ou de registres dans des séries organiques $^{3}$ et la volonté de les maintenir groupés, pour des commodités de recherches et par habitude. Suivant la solution retenue, l'archiviste peut être amené à réserver plusieurs centaines d'emplacement pour une seule sous-série ${ }^{4}$. D'où une suite d'interrogations et des réponses pragmatiques mises en œuvre sur le terrain, en dehors de toute directive officielle et donc de manière non réglementaire. Trois options sont possibles. L'une vise à conserver le cadre de classement coûte que coûte, en maintenant les séries alphanumériques donc, l'autre en fait le deuil en proposant des cotations intersérielles ${ }^{5}$ (annexe 1). L'ultime manière de s'accommoder du système est de ne pas coter l'article mais d'arrêter la cotation au niveau de la série ou de la sous-série organique, en constituant des sections ou subdivisions de sous-

3. Division organique d'un fonds, correspondant à un ensemble de dossiers maintenus groupés parce qu'ils résultent d'une même activité, se rapportent à une même fonction ou à un même sujet, ou revêtent une même forme.

4. Pour prendre un exemple simple, la sous-série $4 \mathrm{Q}$ qui regroupe les fonds des bureaux des hypothèques comprendra au minimum deux séries organiques par bureau et huit sous-séries organiques. Sachant qu'il y a approximativement un bureau d'enregistrement par canton, cela donne une idée du nombre de sous-séries organiques en voie d'accroissement (plusieurs centaines par département).

5. La trace la plus ancienne de cotation continue confirmée à ce jour date de 1'après-première guerre mondiale, mais il est probable qu'il y en ait de plus anciennes. Dans la Drôme, Jacques de Font-Réaulx avait instauré une série continue intersérielle dès 1920, en utilisant la série Z, alors affectée aux documents divers. Le classement continu intersériel est d'ailleurs préconisé depuis 1933 par deux archivistes départementaux Henry Chanteux et Joseph Estienne (Gazette des archives, 1933, p. 88-89). 
séries, permettant ainsi une intégration infinie des versements et une prolifération des espaces réservés ${ }^{6}$.

Des séries d'attente permettant de ranger en continu apparaissent ainsi de manière presque systématique. On sait qu'en 1955, ce classement continu temporaire est en vigueur dans la plupart des départements de l'aveu même de l'inspection générale ${ }^{7}$. Certains vont même jusqu'à imaginer la fin du cadre de classement et la mise en place officielle de nouveaux usages, série continue définitive par exemple. La direction hésite, se montre tolérante ici, décourage $1 a^{8}$. La profession, elle, se sent menacée. Ce sentiment est d'autant plus fort qu'elle doit faire face à une offensive du monde documentaire qui tente de lui imposer ses propres méthodes et à un foisonnement d'initiatives dont certaines semblent bien peu orthodoxes. Ces hésitations et expérimentations durent jusqu'en $1979^{9}$, date de l'officialisation de la série continue « intersérielle », c'est-à-dire confondant tous les dossiers autrefois répartis dans les séries méthodiques, qui marque la victoire d'un type de cotation sur beaucoup d'autres solutions possibles. Quatorze ans après la clôture du cadre de classement au 10 juillet $1940^{10}$, les conditions semblent réunies, aux yeux du directeur Jean Favier et de l'inspecteur général Michel Duchein, pour un changement de culture.

\section{La série continue : une révolution matérielle?}

Avec la création de séries continues, se posent deux questions connexes, celle du récolement permanent et celle du catalogage. Pour tirer les bénéfices du nouveau mode de cotation en termes de gestion de 1'espace, de coûts économiques et d'efficience, il faut pouvoir rechercher les données plus efficacement, identifier les éliminations et produire des instruments de recherche mis à jour en temps réel.

\section{Un changement induit dans les pratiques}

On peut en effet constater que le passage à une cotation continue (sérielle ou intersérielle) s'accompagne d'un changement dans les instruments qui permettent de rechercher les fonds. Les systèmes les plus élémentaires, comme celui de l'Eure, vise à se passer presque totalement de répertoires en poussant la logique de la répartition au bout. Au contraire, le passage à la cotation continue dans le système du cadre de classement suppose de

\section{Cas de 1'Eure.}

7. Léonce Cellier dans La Gazette des archives, $\mathrm{n}^{\mathrm{os}} 17-18$, janvier-juillet 1955, p. 66.

8. Henri Charnier, « Les versements des services des préfectures dans les archives départementales », La Gazette des archives, $2^{\mathrm{e}}$ trimestre 1959 , nouvelle série $\mathrm{n}^{\circ} 26, \mathrm{p} .6-32$.

9. La circulaire AD 79-6 portant instruction sur le traitement des documents des archives départementales postérieurs au 10 juillet 1940 crée la série continue W.

10. Décidée par la circulaire AD 65-29 du 16 décembre 1965 sur la cotation, le classement et le répertoire des séries modernes. 
trouver un moyen de corréler un numéro de rangement avec une localisation intellectuelle, celle de la série alphanumérique.

Les solutions retenues sont de plusieurs ordres. La première vise à perturber le moins possible les habitudes acquises. Sur le bordereau de versement, est indiquée la cotation de l'article (fig. 2). On peut aussi choisir d'ouvrir des registres par série et y recopier scrupuleusement les informations relatives aux articles cotés en continu et virtuellement attachés à une série. Certains services conservent une collection de bordereaux dans des classeurs à feuillets mobiles dans l'ordre des séries pour gérer plus aisément les mises à jour après élimination.

\section{Les fichiers comme solution pérenne}

Dans la plupart des cas, la cotation continue s'accompagne de la tenue de fichiers, soit comme instruments de gestion, soit comme instruments d'orientation, soit comme instruments de recherche (fig. 3). Ces fichiers se révèlent centraux, en particulier pour les services assurant du pré-archivage.

Pour gérer au mieux les éliminables, il convient de tenir deux fichiers : un fichier des éliminables (liste d'éliminables sur fiches) par année d'élimination, permettant d'identifier les documents à détruire et d'optimiser l'espace, et un fichier des versements dans lequel sont insérées des fiches de différentes couleurs que l'on retire au fur et à mesure des éliminations $^{11}$. Ce fichier des versements est d'ailleurs 1'instrument de recherche principal dans les services les plus engagés dans le pré-archivage et la collecte des documents contemporains, même s'il existe parallèlement des collections de bordereaux chronologique et par service. On accède aux informations en cherchant le nom du producteur qui est replacé finement dans sa hiérarchie administrative ${ }^{12}$. Le système n'est vraiment complet qu'avec la mise en place d'un fichier d'indexation, retardé pour cause d'absence de thesaurus. Ce fichiermatières a existé dans quelques cas mais on s'est souvent contenté de fichiers méthodiques (dans 1'ordre des séries du cadre de classement).

Les archivistes ont donc consenti à adopter des outils de travail nouveau (fichiers, classeurs à feuillets mobiles), mais avec un réel retard puisqu'ils ne semblent se répandre qu'après 1945 et ne sont recommandés qu'en $1965^{13}$. Ils n'ont pas, en revanche, utilisé des techniques plus automatisées (cartes perforées par exemple) pourtant en cours dans certains services de documentation. On peut certainement imputer cette réticence à acclimater des technologies nouvelles à une forme de conservatisme de la profession lié à sa formation intellectuelle et à l'image qu'elle a de ses missions. Au demeurant, en 1979, les services

11. Système en cours dans la Drôme ou dans le Cantal.

12. Cas du Loiret.

13. Circulaire AD 65-29 du 16 décembre 1965, déjà citée. 
utilisant des fichiers ou des classeurs avec le degré de raffinement décrit précédemment sont peu nombreux ${ }^{14}$.

\section{La concurrence documentaire}

Ce conservatisme se lit également dans la réaction de la profession aux innovations inspirées d'autres disciplines. Au début des années 1950, des professionnels de la documentation tentent d'investir le terrain des archives par le cheval de Troie de la cotation et du cadre de classement. Une bataille s'engage alors dont les archives en formation sont l'enjeu.

\section{La classification décimale comme nouveau mode d'organisation}

$\mathrm{Au}$ niveau européen, des modes de classification décimale - Dewey ou classification décimale universelle (CDU) - ont été introduits pour la gestion des archives courantes ou historiques dans plusieurs pays : Hongrie, Hollande, Danemark et Belgique. La CDU est également en vigueur au sein d'organismes internationaux, notamment 1'UNESCO qui, en raison de ses liens avec le Conseil international des archives et de son siège parisien, attire plus particulièrement l'attention des Français. En France même, des tentatives d'introduction de ce système pour organiser les archives actives sont envisagées ou menées à un niveau institutionnel - le ministère de l'Intérieur ${ }^{15}$ - ou à un niveau plus local sous l'effet d'initiatives individuelles ou commerciales ${ }^{16}$.

Les partisans de la CDU sont des militants d'un projet qui dépasse les simples questions techniques pour viser à une pensée organisatrice du monde. Par l'instauration d'un système unique, ils prétendent uniformiser au niveau international le traitement des archives. Ils revendiquent avec force leur parti pris organisationnel, mènent des actions de lobbying et rencontrent parfois une audience favorable. La fédération internationale de documentation et le président du comité central de classification, René Dubuc, par ailleurs délégué français, produisent plusieurs documents visant à adapter la classification décimale universelle au domaine des archives en général et à celui des archives des communes en particulier ${ }^{17}$. René Dubuc entreprend dès 1952 différentes actions auprès des professionnels et des décideurs politiques pour faire accepter ce système de classement en France. En 1954, Johan Pieterse, chef du registry à 1'UNESCO, fait paraître dans Archivum un article qui fait grand bruit ${ }^{18}$ sur les bienfaits de 1'application de la CDU au domaine des records. Le Belge Adolf Bucqué

14. Enquête sur 1'application de la circulaire AD 79-6 dans les archives départementales. Arch. nat., site Fontainebleau, 2003/0279/13.

15. « Réunion des archivistes de 1'Est», La Gazette des archives, n 8, juillet 1950, p. 16. Signale 1'édition en cours par le ministère de 1'Intérieur d'une publication « Documentation communale ».

16. Des archives communales ont connu des classements CDU dans la Sarthe par exemple.

17. Arch. nat., $\mathrm{AB}^{\mathrm{XXXI}} 315$.

18. Archivum. Revue internationale des archives, 1954, vol. IV, 1955, p. 179-183. 
tente de lancer en France son système inventé en 1949 et utilisé dans des communes et administrations belges, le Decasepel, et essaie de créer en 1957 une société à Lille pour le diffuser ${ }^{19}$.

\section{Une réaction institutionnelle}

Les archivistes voient avec inquiétude l'irruption de la classification décimale dans 1'organisation des archives courantes et la ressentent comme concurrentielle de leur propre système de classification des archives définitives. La direction des Archives de France bâtit progressivement un argumentaire contre la CDU et défend son propre cadre de classement. Elle rappelle que les missions de contrôle et d'inspection lui reviennent et que : «En aucun cas, la «classification décimale universelle » ne peut donc être introduite dans le classement des archives communales françaises; toute initiative en ce sens irait à l'encontre des règlements en vigueur. ${ }^{20}$

Dans un courrier au directeur des archives départementales de la Sarthe, la réponse est plus nuancée. La direction reconnait qu'il faut en fait distinguer les fonds clos et les fonds ouverts, c'est-à-dire les archives définitives et les archives courantes. «[...] les dossiers d'administration courante peuvent être classés selon le cadre que chaque service responsable trouve le meilleur et le plus commode, à condition, bien entendu, que ce cadre s'adapte aisément au cadre alphabétique officiel au moment de 1'archivage définitif ${ }^{21}$ ». Elle ajoute ne pas souhaiter voir s'étendre ce système.

À 1'argument international, la direction répond que l'idée de produire par l'adoption de ce système une uniformisation internationale du traitement des archives est bâtie sur une illusion. La matière même des archives est fluctuante pour chaque pays et nécessite donc des adaptations à l'esprit des nations ${ }^{22}$.

\section{Un aiguillon pour la réflexion}

Face à cette offensive documentaire, la profession engage une réflexion sur ses pratiques de classification. En 1952, 1'Association des archivistes français met à l'étude la question des méthodes de classement ${ }^{23}$. Dans le questionnaire élaboré et mis en discussion lors des réunions régionales puis débattu en assemblée générale, les questions I, II, VI, VII, IX, X et XI (annexe 2) concernent directement le choix d'un plan de classification. Dans la formulation, les rédacteurs opposent le principe de respect des fonds et la répartition selon la CDU (question VII) car ils ne distinguent pas le traitement des archives courantes de celui des archives définitives. Notons néanmoins que la question de la compatibilité entre respect des

19. Lettre du 17 mai 1957. Arch. nat., $\mathrm{AB}^{\mathrm{XxXI}} 315$.

20. Avis du 24 juin 1957. Arch. nat., $A B$ XXXI 315.

21. Lettre du 15 septembre 1958. Arch. nat., $A B^{\text {XXXI }} 315$.

22. Rapport sur le cadre de classement des archives communales du 7 janvier 1953, 7 feuillets. Arch. nat., $\mathrm{AB}^{\mathrm{XXXI}} 315$.

23. «Question mise à l'étude avant la prochaine assemblée générale. Méthodes de classement », La Gazette des archives, $\mathrm{n}^{\circ} 11$, janvier 1952 , p. 7-9. 
fonds et CDU est réelle. Dans le cas d'un enregistrement centralisé, la provenance n'est pas forcément strictement respectée lors de la cotation CDU.

Henri Charnier, archiviste départemental et futur président de l'Association des archivistes français, se faisant l'écho de ses collègues, dénonce des «systèmes de classement d'autant plus à la mode qu'ils sont incompatibles avec celui des bureaux, et [...] ne respectent même pas toujours le cadre d'activité des bureaux. [...] Mais un beau jour un classement décimal quelconque pourrait bien être imposé à l'échelon national, qui ne serait pas un simple habillage de nos séries [...] Ainsi triomphent et la confusion de l'empirisme avec le désordre et toutes les séductions des codes plus ou moins chiffrés et "progressions" plus ou moins

alphabétiques. " ${ }^{24} \mathrm{Ce}$ faisant, il ne pointe pas tant l'utilisation de tel ou tel système que l'inertie des Archives face à 1'introduction de nouvelles méthodes administratives et de management.

\section{Les méthodes modernes : le self-indexing system}

Si les classifications CDU ou Dewey sont condamnées sans appel, il existe d'autres tentatives classificatoires fondées sur des systèmes décimaux, sur des divisions sous forme d'arborescence hiérarchique par tranche maximale de 10 unités.

\section{Une expérience originale : la grille Pérotin}

La grille Pérotin est un cadre de répartition des fonds mis au point pour les Archives de la Seine par son directeur Yves Pérotin. Yves Pérotin est un chartiste d'un profil particulier. Il a été secrétaire de la table ronde des archives donc en contact avec des archivistes du monde entier et archiviste à l'Office européen des Nations-Unies à Genève. Considéré comme l'introducteur en France de la théorie des trois âges, il est connu pour ses études sur le Records management aux États-Unis et en Angleterre ${ }^{25}$. Aux archives de Paris dont la particularité est de recevoir des fonds communaux et départementaux et donc d'utiliser les deux cadres de classement afférents, à côté d'autres expérimentations toutes orientées vers les archives contemporaines, il teste, à partir de 1960, un système de self-indexing de la série d'attente, système qui est la pierre angulaire de la gestion des documents intermédiaires jusqu'à son abandon commandé par la mise en place réglementaire de la série $\mathrm{W}$ à partir de 1980.

La grille Pérotin met en œuvre une codification des services tels qu'ils sont connus par les bottins administratifs de 1959. Elle permet de répartir les bordereaux de versement qui prennent place dans des hamacs ou dossiers suspendus, avec un code couleur par domaine. Elle utilise un système décimal, avec 9 domaines portant un chiffre de 1 à 9 (annexe 3), subdivisés jusqu'à six niveaux. C'est un subtil mélange entre l'identification plus ou moins

24. «Les versements des services des préfectures dans les archives départementales », article cité, p. 30.

25. Le Records Management et l'administration américaine. Rapport de mission adressé au préfet de la Seine par Yves Pérotin, dact., 61-VI p. «Le "Records Management"' et l'administration anglaise des Archives », La Gazette des archives, $1^{\text {er }}$ trimestre 1964, nouvelle série, ${ }^{\circ}$ 44, p. 5-17. 
précise du producteur du fonds et l'indication d'une matière. Il convient de noter que c'est l'indication du producteur qui est structurante et que, quels que soient le numéro choisi et son degré de précision, le producteur est clairement identifié. Il importe aussi de relever combien ce cadre est une photographie à un moment donné d'un organigramme. La classification fonctionne comme une indexation du bordereau, elle ne constitue pas la cote en elle-même puisqu'elle est couplée à une autre cotation en continu comportant deux chiffres qui indiquent 1'année, complétés par un numéro de quantième. Ainsi, le bordereau 101/76/1 représente le $1^{\text {er }}$ versement du cabinet du préfet (101) de l'année $1976^{26}$.

L'objectif principal de la grille est d'identifier la provenance très précisément. Il s'agit de codifier le service. On est donc dans l'application stricte du respect des fonds et de la provenance. C'est un cadre temporaire permettant d'enregistrer les versements au fur et à mesure des entrées, afin d'en soustraire les documents à éliminer. Les documents définitifs ont vocation à être transférés dans les séries du cadre de classement réglementaire.

\section{Une réception mitigée}

Dès avril 1959, Yves Pérotin avertit l'inspection de son projet d'établissement d'un cadre de numérotation décimal réservé aux services. Le directeur lui demande instamment d'attendre $^{27}$, ce qui ne le décourage absolument pas. Il faut dire qu'il reçoit par ailleurs le soutien ferme de son autorité administrative de tutelle dans le département de la Seine. L'expérience est considérée avec méfiance. On en trouve trace dans les pages du Mamuel d'archivistique auquel Yves Pérotin a collaboré dans les années 1963-1964. Il y expose ses théories sur les cadres de classement, propose d'introduire le système décimal dans les cadres réglementaires existants. L'association des archivistes français accepte de donner de la visibilité à ces essais - Yves Pérotin est un professionnel à la compétence internationalement reconnue dans le domaine des archives administratives. Mais elle le fait en lui marquant une forme de défiance, montrant là qu'une bataille entre anciens et modernes s'est jouée, et en faisant précéder son essai d'adaptation des cadres de classement d'un avertissement ${ }^{28}$ : «il s'agit d'une étude de caractère théorique qui ne saurait en aucune façon conduire à la nonobservation des dispositions réglementaires ».

\section{Des objectifs détournés}

Au fil du temps, les difficultés dans la mise en œuvre de la codification Pérotin se sont multipliées : complexité des cotes, problème de gestion de 1'espace non résolu, difficulté d'utilisation par l'ensemble des agents de l'arborescence hiérarchique. Le problème principal est venu de l'installation dans le temps d'une grille qui était pensée comme éphémère. Parce

26. Il était prévu de compléter le dispositif d'un fichier à cartes à perforations marginales. Rapport annuel. $1^{\text {er }}$ juillet 1960-30 juin 1961, 13 p. Archives de Paris, bibl. 34 Db 75/25.

27. Arch. nat., $\mathrm{AB}^{\mathrm{XXXIC}} 248$.

28. Association des archivistes français, direction des Archives de France, Manuel d'archivistique. Théorie et pratique des archives en France, Paris, Archives nationales, 1991, réimpression de 1'édition de 1970, 805 p., p.717-723. 
qu'elle collait strictement aux organigrammes afin d'identifier au mieux la provenance, la codification devait évoluer avec les services. Les révisions n'ont pas eu lieu, alors que les structures administratives dans la région parisienne ont profondément muté. Au final, les versements « Pérotin » ont été recotés et inclus dans la série réglementaire W.

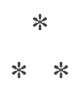

Dans les années cinquante et soixante, les archivistes ont été dans l'obligation d'imaginer des systèmes fatalement complexes pour résoudre des problèmes pour lesquels les traitements automatisés et les logiciels documentaires permettent aujourd'hui de concevoir d'autres solutions. Ils ont découvert aussi d'autres modes de réflexion, d'autres conceptions. La théorie des trois âges a profondément ébranlé les convictions et a mis plusieurs années avant de pénétrer vraiment les conceptions communes. Interroger la question des modes de classification à ce moment précis nous permet de confirmer certains habitus professionnels et d'évaluer les relations entre principes théoriques et techniques professionnelles.

On ne peut que constater un attachement extrêmement fort des archivistes français à leur mode de classification alphanumérique. Il est vrai qu'il est singulier dans le monde. Beaucoup de pays ont des systèmes de répartition des fonds sous la forme d'Archives Group ou de Records Group (groupe de fonds). Mais, « le groupement de fonds le plus connu et peut-être le plus ancien est le classement par séries des Archives françaises, la plus vaste entreprise de ce genre. ${ }^{29} \gg$ Cet attachement s'explique par le fait que le cadre de classement a une fonction identitaire. On est archiviste parce qu'on manie un cadre de classement ${ }^{30}$. Le cadre de classement valorise une compétence propre au métier, un savoir-faire particulier qui confère la légitimité professionnelle.

L'interrogation sur la classification donne également un point de vue sur l'intégration des concepts par les praticiens. Le respect des fonds, dans toutes ses composantes, est parfaitement connu de la profession des archivistes dans les années cinquante et soixante. $\mathrm{Si}$ on regarde avec attention les diverses cotations mis en place pour résoudre les problèmes d'intercalation, si on étudie les réponses données par les archivistes départementaux à l'enquête menée par la direction des Archives de France suite à la création de la série W et les difficultés qu'elle suscite, on s'aperçoit que l'échelon jugé pertinent tant pour le classement que pour les recherches n'est pas le fonds mais la série organique. D'ailleurs, on peut noter la pauvreté des réflexions sur la codification des services, en dehors de la grille Pérotin et un peu plus tard de celle mis au point au centre des archives contemporaines à Fontainebleau. À

29. Rapport du Hongrois Antal Szedö sur les méthodes modernes de classement, Archivum. Revue internationale des archives, vol XIV, 1964, p. 62.

30. Lorsqu'à l'occasion du dixième anniversaire de la formation d'Angers en 2004, j'avais examiné les travaux réalisés par des étudiants dans des structures où il n'y avait pas d'organisation réglementaire des archives, j'avais été frappée du recours presque naturel au cadre alphanumérique. 
1'inverse, la perception du niveau fonds est encore très large. On se contenterait facilement de l'identification globale de l'organisme producteur (la préfecture par exemple) sans descendre plus précisément dans l'organigramme. Ce constat mériterait d'être affiné par l'étude des classements eux-mêmes : les fonds sont-ils identifiés dans les séries? Les sous-fonds dans les fonds?

Enfin, est-il raisonnable d'affirmer la fin des cadres de classement ? Ce n'est pas si sûr. Certes, la série W a été instaurée en 1979. Mais pas entièrement : certaines séries organiques ont été laissées en accroissement, notamment les plus consultées comme l'état civil et les minutes notariales. Depuis, le cadre de classement a fait l'objet d'une refonte en 1998 qui a notamment assoupli la coupure chronologique de 1940 et a créé de nouvelles séries. Par ailleurs, on peut noter çà et là des réflexions sur la présentation des Archives Group à la française et le développement des archives sur le web est certainement une occasion à saisir. Le site de la Vendée, dans la version mise au point en 2008, propose au public un nouveau mode d'entrée dans les fonds en revisitant le cadre de classement départemental de 1841 (fig. 4). C'est une répartition en Archives Group qui partage avec le cadre de classement d'être un compromis entre principe de provenance et principe de pertinence, conservant ainsi une forme de spécificité française. 


\section{Illustrations}

Fig. 1. Extraits des cadres de classement de 1841, 1965 et 1998. Série Q-Domaines, enregistrement, hypothèques.

Fig. 2. Bordereau de versement indiquant la sous-série de destination des articles.

Fig. 3. Typologie des fichiers liés à la mise en place d'une série continue

Fig. 4. Copies d'écran du site des archives départementales de la Vendée

(http://archives.vendee.fr/, consulté le 25 février 2009). 


\begin{tabular}{|c|c|}
\hline 1841 & Q. Domaines....... $\left\{\begin{array}{l}\text { Domaines nationaux. } \\
\text { Administration et contentieux des domaines. } \\
\text { Biens communaux vendus en } 1813 \text { au profit de la } \\
\text { caisse d'amortissement. }\end{array}\right.$ \\
\hline 1965 & $\begin{array}{l}\text { SERIE Q (I790 - I940) } \\
\text { 1. Domaines nationaux (époque de la Révolution française, y } \\
\text { compris la liquidation des domaines nationaux, les restitu- } \\
\text { tions et indemnités ( } 1 \text { ). } \\
\text { 2. Doinaine (période postérieure à la Révolution française) (2). } \\
\text { 3. Enregistrement et tirubre (3). } \\
\text { 4. Hypothèques (4). } \\
\text { (1) Ordre proposé : a) fonds de l'administration départementale : } \\
\text { Direction, Administration centrale, Préfecture, puis Districts, } \\
\text { dans l'ordre des districts ; b) les fonds de l'administration } \\
\text { des Domaines : direction départementale, puis classement par } \\
\text { bureaux. Cf. Carille Bluch ; Ie classement de la série Q des } \\
\text { Archives départementales, dans Ia Révolution française, } \\
\text { no 23-24, mai-juin I9I6, p. l-32). }\end{array}$ \\
\hline 1998 & 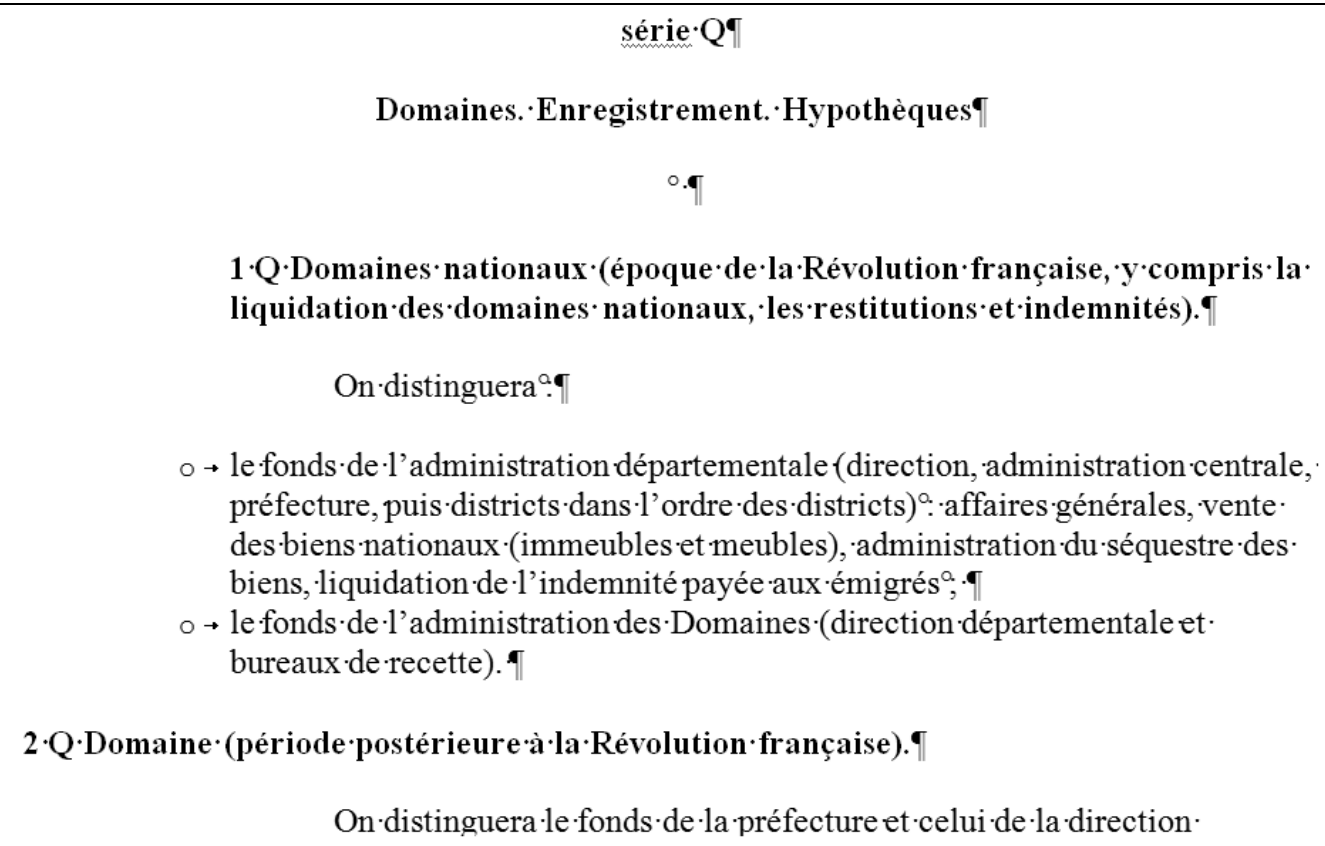 \\
\hline
\end{tabular}

Fig. 1. Extraits des cadres de classement de 1841, 1965 et 1998. Série Q Domaines, enregistrement, hypothèques Retour au texte, cliquez ici 


\begin{tabular}{l|l}
\hline $\begin{array}{l}\text { PREFECIURE } \\
\text { Direction des affaires financières } \\
\text { 3e bureau }\end{array}$ & Classé en \\
\hline $\begin{array}{l}\text { Taxes d'apprentissage } \\
\text { Caisses départementales scolaires }\end{array}$ & $15 \mathrm{M} 111$ \\
\hline Equipement sportif socio-éducatifs (par commune) & 506 \\
\hline Accidents scolaires & $505^{12}$ \\
\hline
\end{tabular}

Fig. 2. Bordereau de versement indiquant la sous-série de destination des articles Retour au texte, cliquez ici
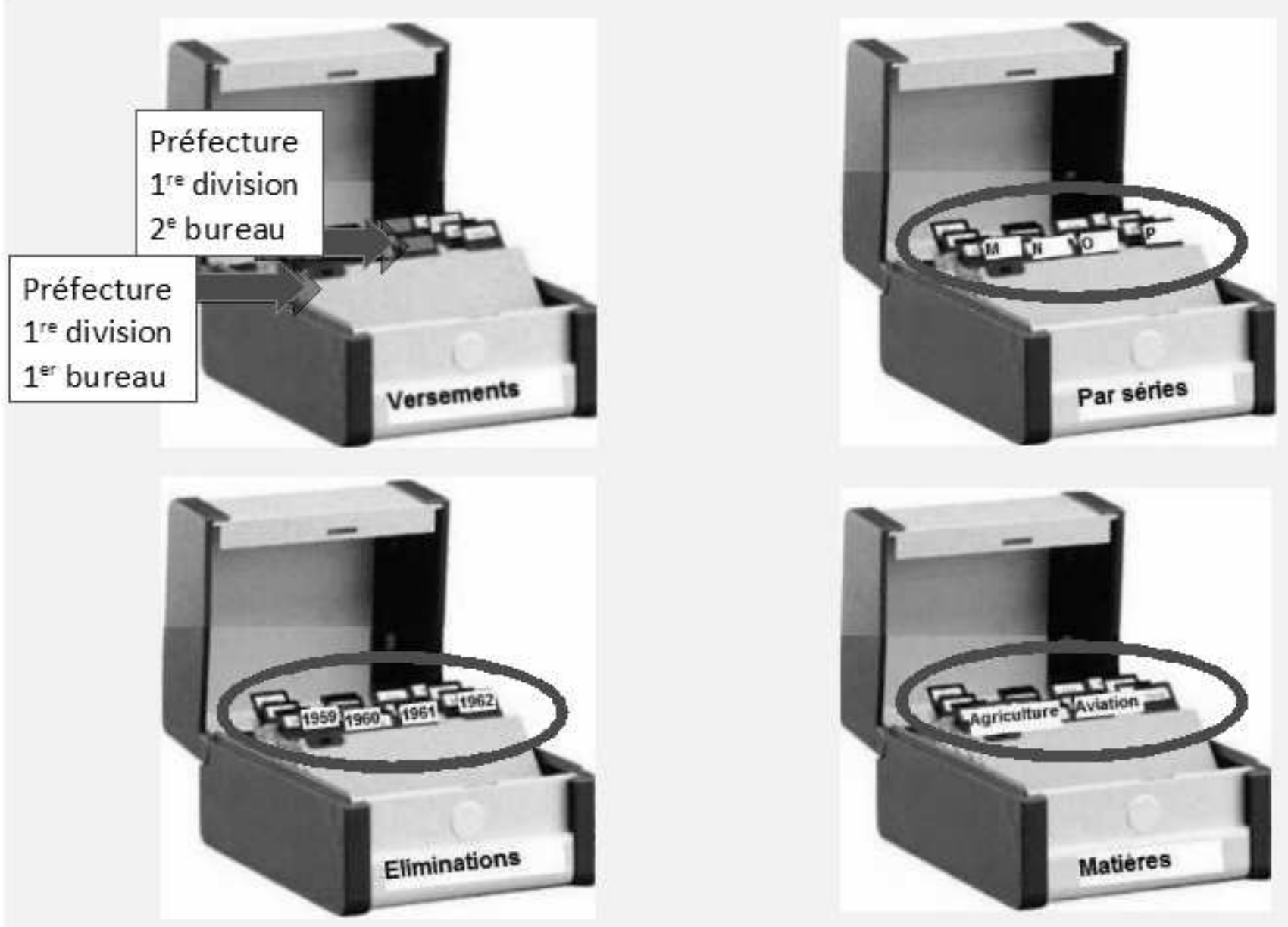

Fig. 3. Typologie des fichiers liés à la mise en place d'une série continue Retour au texte, cliquez ici 
Vous êtes ici : Accueil > Inventaires et cataloques en ligne

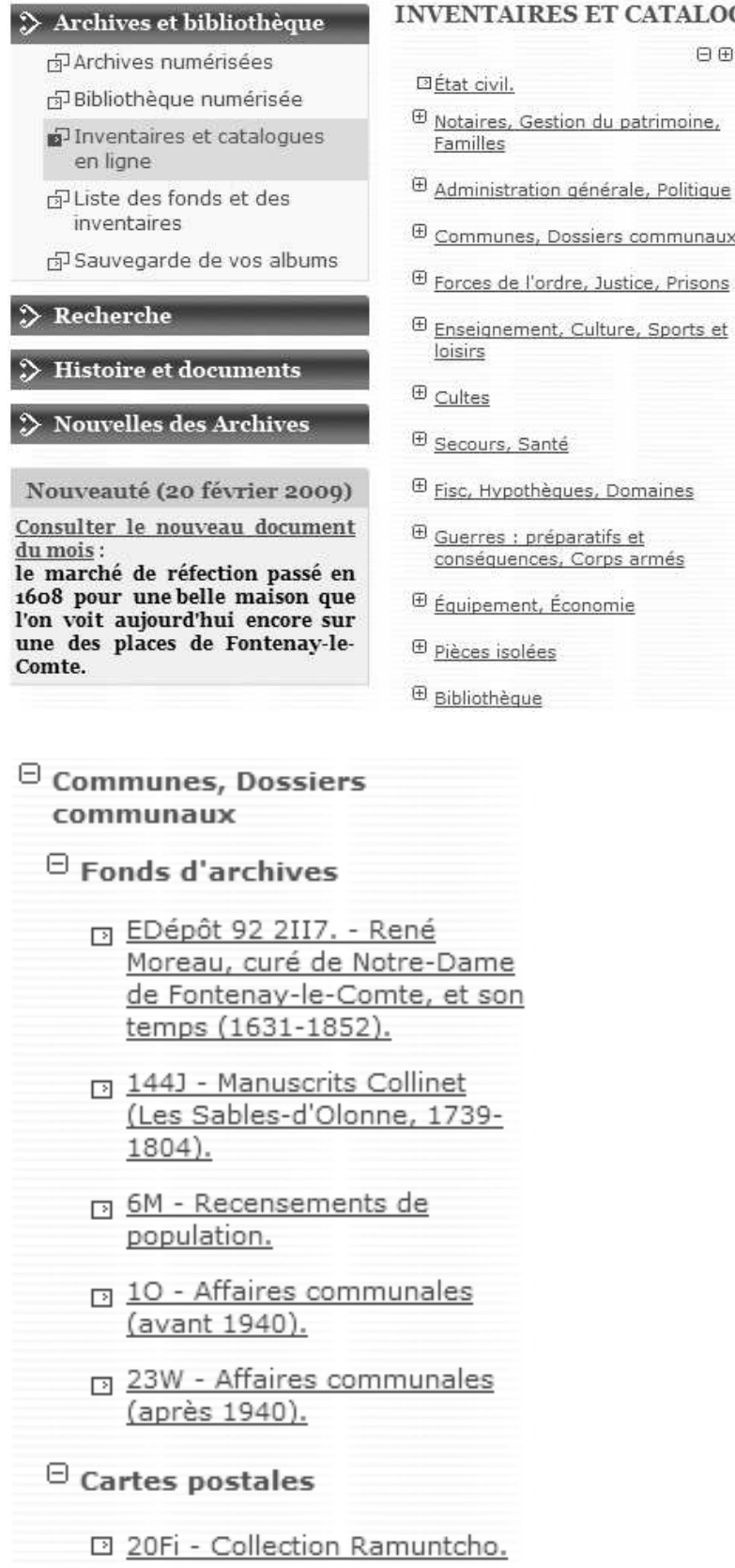

Nouveauté (20 février 2009) Consulter le nouveau document

$\checkmark$ 20Fi - Collection Ramuntcho.

$\boxplus$ Notaires, Gestion du patrimoine, Familles

$\boxplus$ Administration qénérale, Politique

$\boxplus$ Communes, Dossiers communaux

$\boxplus$ Forces de l'ordre, Justice, Prisons

$\boxplus$ Enseianement, Culture, Sports et loisirs

$\boxplus$ Cultes

$\boxplus$ Secours, Santé

$\boxplus$ Fisc, Hypothèques, Domaines

$\boxplus$ Guerres: préparatifs et conséquences, Corps armés

$\boxplus$ Équipement, Économie

$\boxplus$ Pièces isolées

$\boxplus$ Bibliothèque

\section{INVENTAIRES ET CATALOGUES EN LIGNE}

\section{Présentation}

Un cadre thématique de 13 rubriques présente les inventaires d'archives, les catalogues de notices bibliographiques ou de documents iconographiques.

Pour déployer l'une d'elles, cliquez dessus.

Pour consulter un inventaire électronique, cliquez sur son titre.

Tous les inventaires ne sont pas encore disponibles en ligne. Pour connaître l'étendue des ressources des Archives de la Vendée, consulter le menu Liste des fonds et des inventaires.

Les dernières mises en ligne sont annoncées dans les Nouvelles des Archives. $\boxminus$ Plans d'édifices publics et privés

$\checkmark$ Communes de L'Aiquillonsur-Mer à Givrand.

$\checkmark$ Communes du Givre à SaintAvaugourd-des-Landes.

$\checkmark$ Communes de Saint-Benoistsur-Mer à Xanton-Chassenon.

$\boxminus$ Photographies aériennes

$71 \mathrm{Fi}$ - Fonds LAPIE (19551959).

$\boxminus$ Travaux d'érudits

7]7-18 - Travaux de P. Prouzeau sur Velluire et sa réqion.

134] - Travaux de J. Vincent (principalement sur Les Herbiers).

Fig. 4. Copies d'écran du site des archives départementales de la Vendée (http://archives.vendee.fr/, consulté le 25 février 2009) Retour au texte, cliquez ici 


\section{Annexes}

Tableau 1. Exemple de cotation aménageant le cadre de classement pour intercalation et prenant en compte tous les niveaux de description

Tableau 2. Exemple de cotation aménageant le cadre de classement pour intercalation ne prenant pas en compte le niveau de la série organique.

Tableau 3. Exemple de cotation sérielle partiellement continue, identifiant le fonds .......... 19

Tableau 4. Autres exemples de cotation sérielle continue .................................................... 18

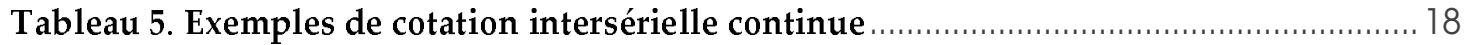

Annexe 2 : Question mise à l'étude par l'association des archivistes français en 1953 ... 19

Annexe 3 : Extrait de la grille Pérotin 
Annexe 1 : Typologie des cotations mises en œuvre dans les services avant 1979 (quatre tableaux)

$\underline{\text { Retour au texte, cliquez ic }}$

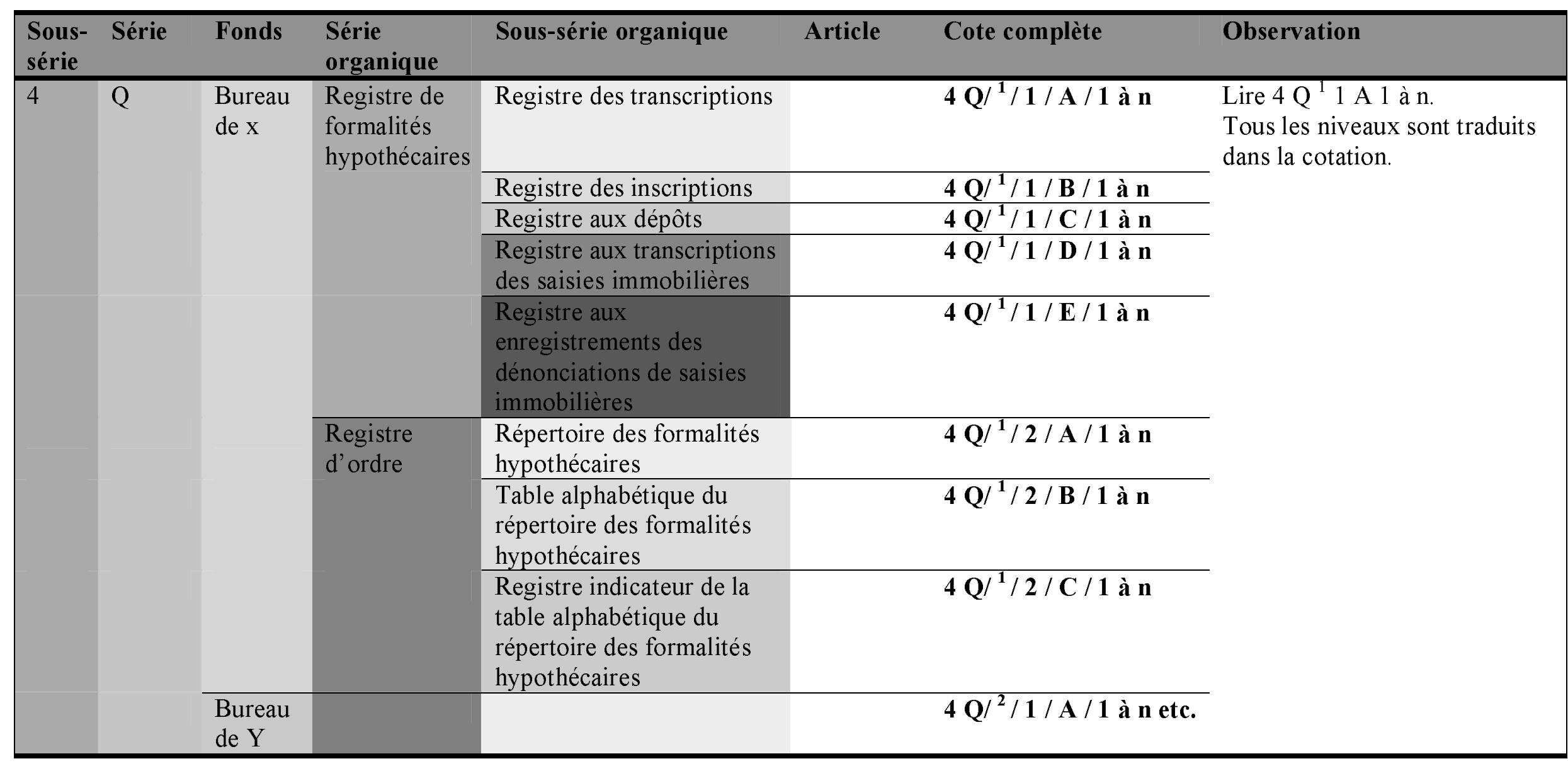

Tableau 1. Exemple de cotation aménageant le cadre de classement pour intercalation et prenant en compte tous les niveaux de description 


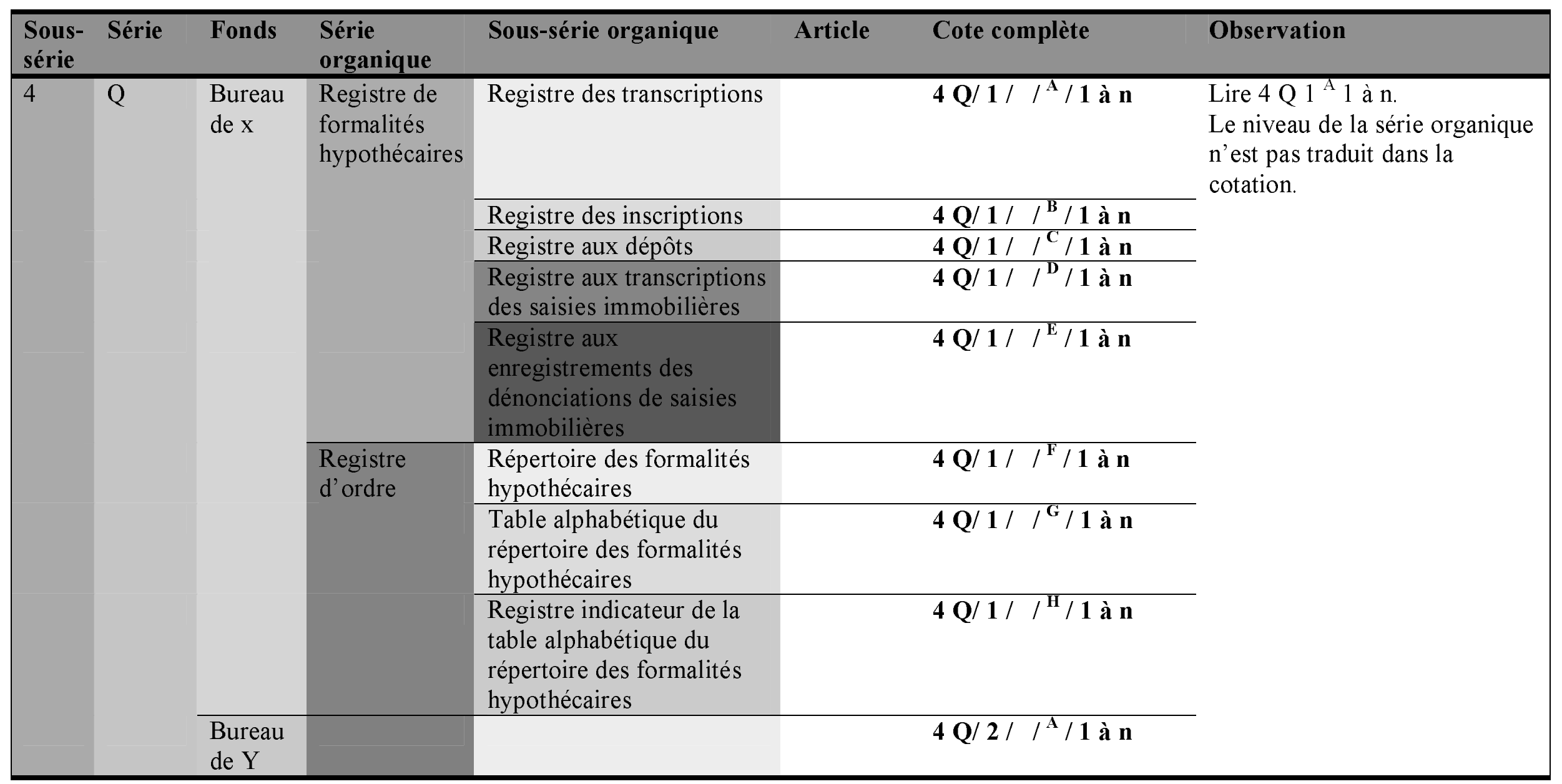

Tableau 2. Exemple de cotation aménageant le cadre de classement pour intercalation ne prenant pas en compte le niveau de la série organique 


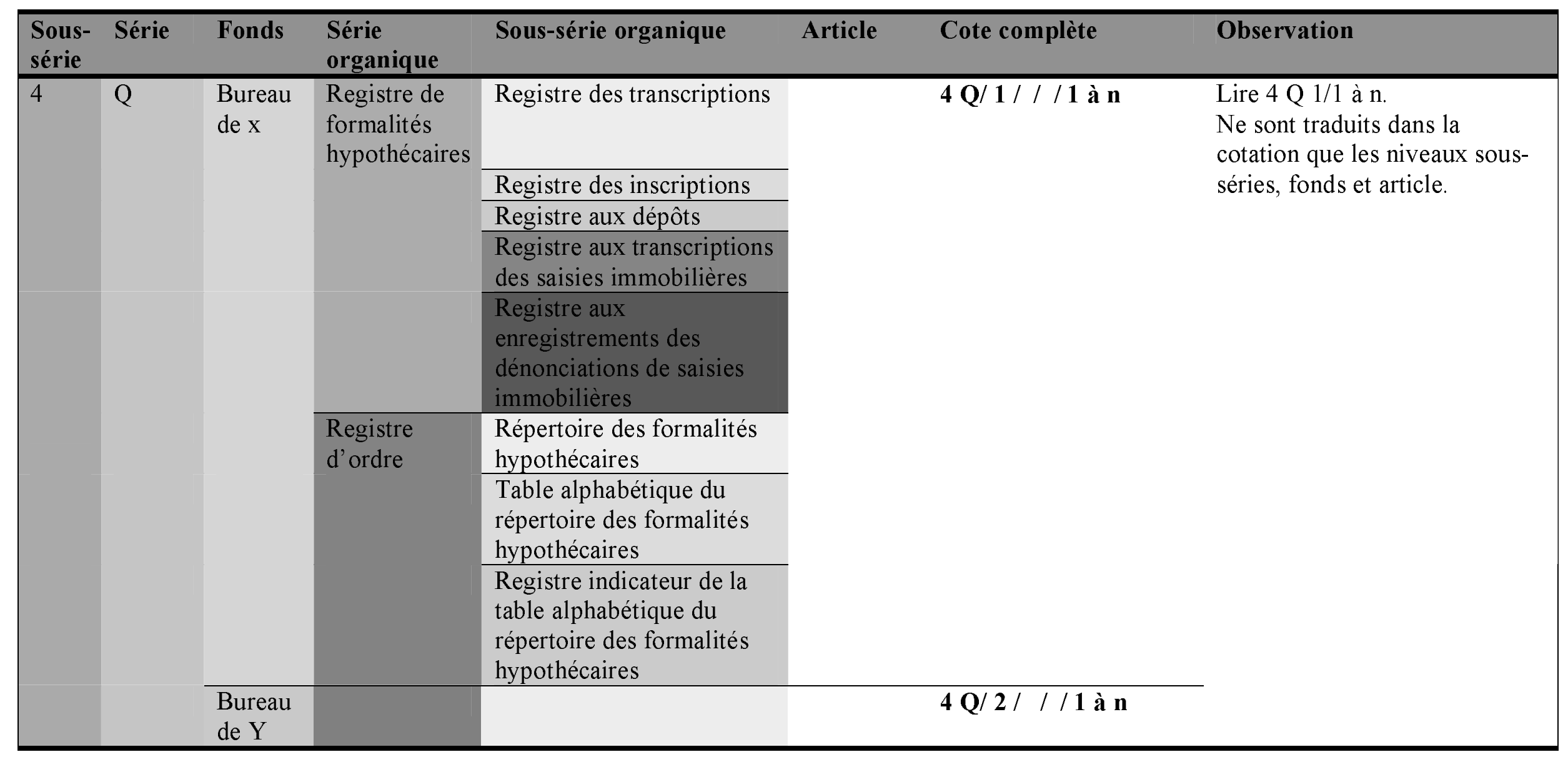

Tableau 3. Exemple de cotation sérielle partiellement continue, identifiant le fonds 


\begin{tabular}{|lll|}
\hline Caractéristiques de la cotation & Type de cotes généré & Interprétation \\
\hline Cotation continue par versement & 4 Q 1/ 1 à n & $\begin{array}{l}1^{\text {er }} \text { versement entré et intégré à la sous-série 4 Q } \\
2^{\text {e }} \text { versement entré et intégré à la sous-série 4 Q etc. }\end{array}$ \\
\hline $\begin{array}{l}\text { Numérotation entièrement continue sans } \\
\text { identification du versement ou du fonds }\end{array}$ & 4 Q 1 à n & \\
\hline
\end{tabular}

Tableau 4. Autres exemples de cotation sérielle continue

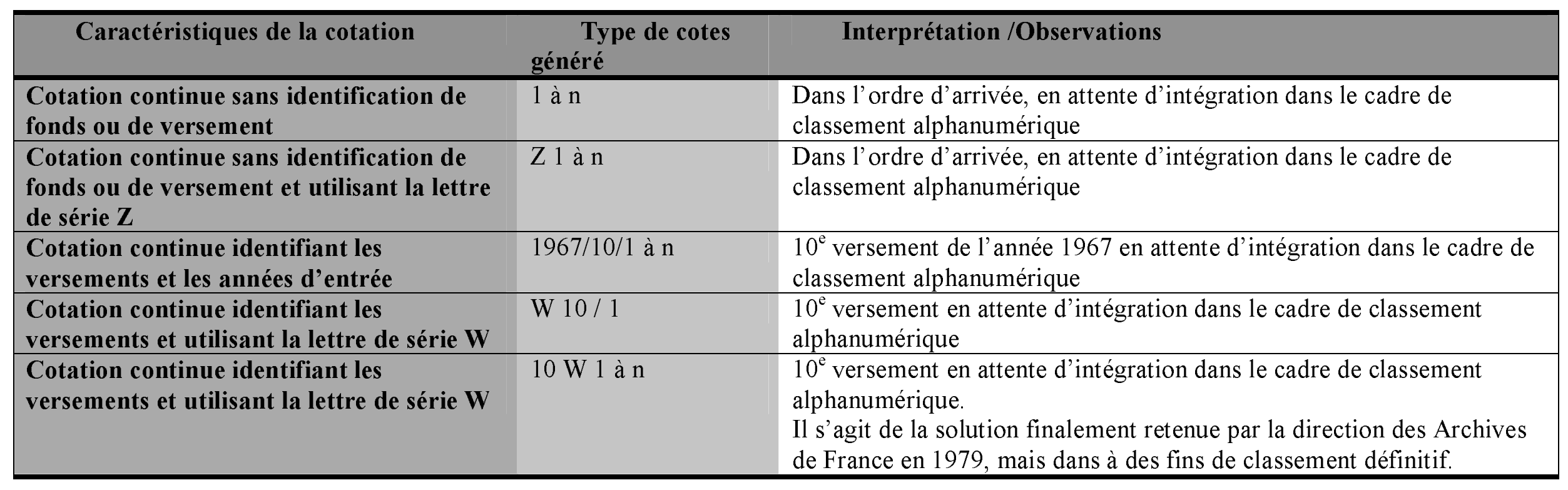

\section{Tableau 5. Exemples de cotation intersérielle continue}

$\underline{\text { Retour au texte, cliquez ici }}$ 


\section{Annexe 2: Question mise à l'étude par l'association des archivistes français en 1953}

$\underline{\text { Retour au texte, cliquez ici }}$

I. Estimez-vous encore valables les principes directeurs du cadre de classement du règlement des Archives départementales de 1841 :

On demande aux adhérents de se prononcer sur trois parti-pris inhérents au principe du cadre de classement, à savoir la répartition en séries anciennes (documents antérieurs à la Révolution), séries révolutionnaires (1790-1800) et séries modernes (à partir de 1800), le « classement méthodique par grands services », l'affectation immédiate des versements à une série de ce cadre.

II. Croyez-vous qu'il y aurait intérêt à introduire un nouveau système de classification à compter d'une date à déterminer $[\ldots]$ ?

VI. Les dossiers à conserver indéfiniment devront-ils être traités comme les livres d'une bibliothèque sans tenir compte de leur contenu et rangés systématiquement les uns à la suite des autres [...] ou bien seront-ils répartis suivant un classement méthodique par service versant $[\ldots]$ ?

VII. Le principe du respect des fonds devra-t-il être intégralement maintenu ou cherchera-t-on à lui substituer un classement méthodique par matières qui pourrait-être la classification décimale universelle ou un système s'en inspirant?

IX. Le classement méthodique par services ne comporte-t-il pas de graves inconvénients du fait des changements d'attributions des services, de la création de nouveaux services, de la suppression de certains d'entre eux ?

$\mathrm{X}$. Êtes-vous favorables à l'adoption par les services versants du système de classement en vigueur dans les archives départementales [...]?

XI. Si vous pensez qu'une telle réforme (classification décimale universelle ou système équivalent) bouleverserait trop profondément les règles admises jusqu'à maintenant, croyez-vous cependant qu'il y aurait lieu de modifier le cadre ou les méthodes de classement actuels?

Extrait de la «Question mise à l'étude avant la prochaine assemblée générale. Méthodes de classement », La Gazette des archives, n 11, janvier 1952, p. 7-9.

Retour au texte, cliquez ici 


\section{Annexe 3 : Extrait de la grille Pérotin}

$\underline{\text { Retour au texte, cliquez ici }}$

\section{Administration générale}

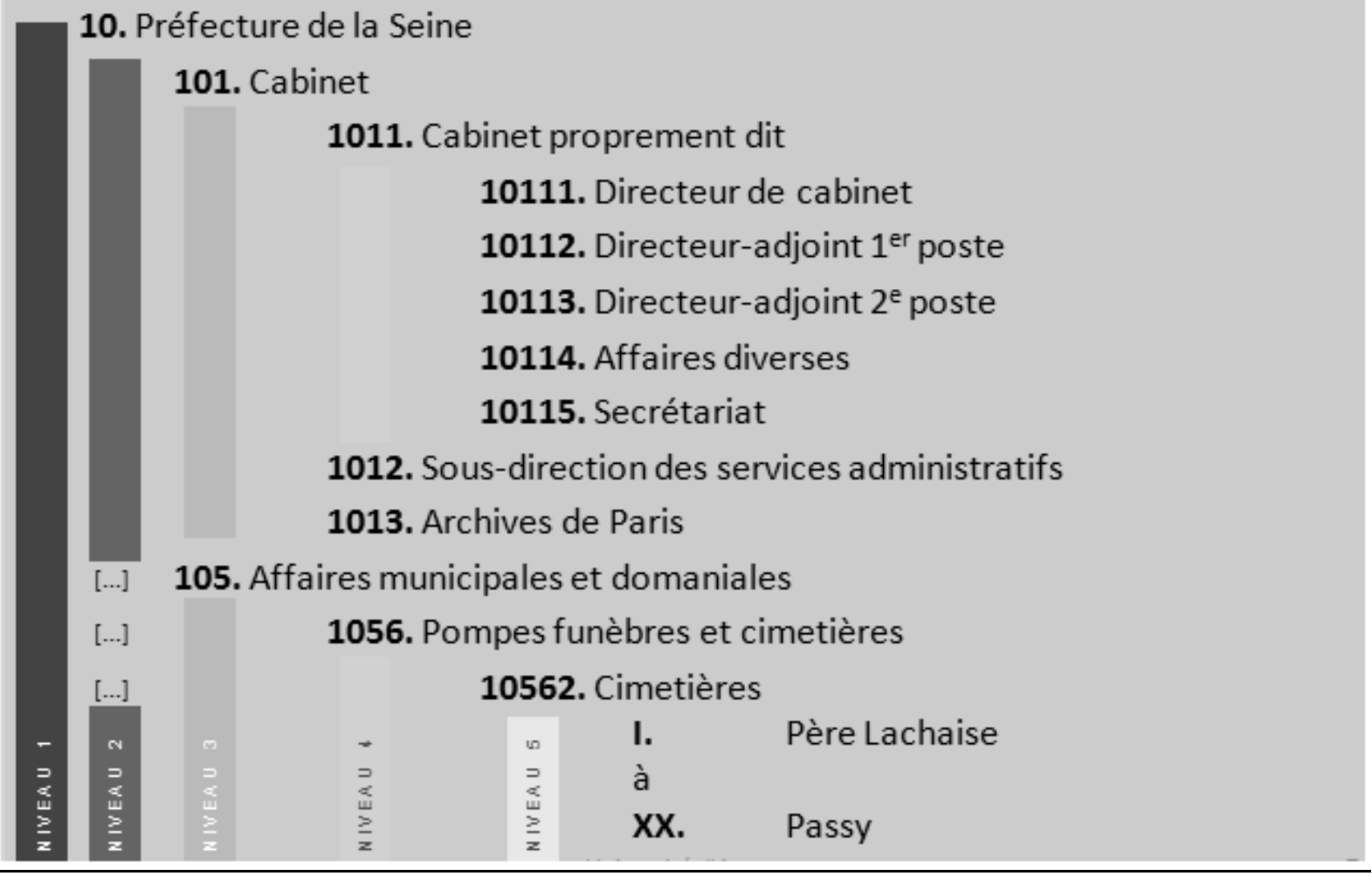

Retour au texte, cliquez ici 